\title{
Lectura académica en profesores de la carrera de psicología de una universidad privada de Arequipa, Perú
}

\author{
Academic reading in psychology career professors from a private \\ university in Arequipa, Peru
}

\section{Walter L. Arias Gallegos ${ }^{1}$}

\section{Revista \\ Educación y Sociedad}

Citar como: Arias Gallegos, W. L. (2021). Lectura académica en profesores de la carrera de psicología de una universidad privada de Arequipa, Perú. Revista Educación y Sociedad, 2(4), 28-34. https://doi.org/10.53940/reys.v2i4.72

Artículo recibido: 15-09-2021 Artículo aprobado: 05-11-2021 Arbitrado por pares

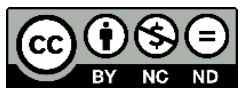

\section{ACEES}

\section{Resumen}

Se describen las fuentes académicas consultadas por los profesores de psicología de una universidad de la ciudad de Arequipa para preparar sus cátedras o mantenerse actualizados. Este estudio descriptivo transversal contó con una muestra de 28 profesores universitarios a quienes se les aplicó una ficha de datos ad hoc, sobre la lectura de libros y la consulta de revistas especializadas. Se encontró que si bien un 94.4\% de profesores lee libros un $39.3 \%$ desconoce revistas especializadas; pero solo se encontraron diferencias significativas en la consulta de revistas en función de la experiencia en investigación que reportaron los profesores evaluados.

Palabras clave: lectura, universidad, docencia universitaria, actividad académica

\section{Abstract}

The academic sources consulted by psychology professors of a university in the city of Arequipa to prepare their lectures or to keep themselves updated are described. This descriptive cross-sectional study included a sample of 28 university professors to whom an ad hoc data sheet was applied, on the reading of books and consultation of specialized journals. It was found that although $94.4 \%$ of professors read books, $39.3 \%$ were unaware of specialized journals; but significant differences were only found in the consultation of journals according to the research experience reported by the professors evaluated.

Key words: reading, university, university teaching, academic activity 


\section{Introducción}

La lectura ha favorecido, a lo largo del tiempo, el desarrollo científico y el progreso social de la humanidad (Cavallo y Chartier, 2011). Por lo tanto, e independientemente del tipo que se trate o de su finalidad, es un medio de transmisión de la cultura (León, 2021). En el ámbito académico, la lectura es una actividad indispensable sobre la que reposan las actividades de enseñanza e investigación, y que debe llevarse a cabo de forma crítica, permanente y especializada (Arias, 2013).

En el Perú, sin embargo, la lectura es un aspecto relegado en todos los niveles formativos ya que según diversos informes como las pruebas PISA los escolares peruanos se ubican en los últimos lugares de comprensión lectora entre los países de la región (Guadalupe y Villanueva, 2013). En este marco, las cifras de analfabetismo alcanzan a aproximadamente al 12\% de la población; siendo esta situación mayormente representada en las mujeres y en los habitantes de zonas rurales de la sierra y la Amazonía (Carrillo y Cuenca, 2019). Al mismo tiempo, se ha mencionado que entre la población alfabetizada el principal problema es el de la comprensión ya que hasta un $60 \%$ no comprende lo que lee; hecho que configura la condición de analfabetos funcionales (Velarde y Canales, 2008).

$Y$ es que la lectura es una actividad compleja que demanda del uso de procesos cognitivos y neuropsicológicos que se forman desde la niñez (Deheane, 2015); amén del empleo de estrategias de enseñanza pertinentes, que apuntan hacia la adquisición del sentido inferencial de los textos leídos (Pradelli, 2013). En el caso de la enseñanza universitaria, se requiere de una alfabetización académica que les permita a los estudiantes vincularse con lecturas especializadas con alto nivel de complejidad (Cisneros et al., 2013). Pero diversos estudios en Arequipa, ciudad ubicada al sur del Perú, han demostrado que los estudiantes universitarios leen poco, leen lento (Arias y Justo, 2012) y no poseen hábitos de estudio adecuados (Montes, 2012).

Más específicamente en la carrera de psicología, se trata de una especialidad que requiere de una gran cantidad de lectura académica para desarrollar un pensamiento crítico y reflexivo, orientada hacia una sólida formación para el ejercicio profesional del psicólogo, sobre la base de evidencia científica (Álvarez et al., 2021). Sin embargo, estudios previos en la ciudad de Arequipa, han reportado que los estudiantes de psicología no poseen habilidades metacognitivas que les permitan autorregular su aprendizaje (Arias et al., 2014) y su motivación de logro académico es baja (Arias et al., 2018). Esta situación, que obedece a deficiencias del sistema universitario en el Perú (Arias, 2013), es agravada por factores culturales. Así, es revelador comprobar que, en un amplio estudio desarrollado en ciudadanos de la capital, lo último que contribuye a la felicidad de los peruanos es la búsqueda de conocimiento (Alarcón, 2002).

Ahora bien, otro factor íntimamente vinculado con la carencia de habilidades académicas de los estudiantes de psicología en relación con la lectura es la falta de preparación académica de la mayoría de sus profesores universitarios (Arias, 2013); y en donde queda evidenciada su escasa motivación por investigar y actualizarse (Merino-Soto y Salas-Blas, 2016). Es decir, que los profesores universitarios no suelen publicar en revistas científicas ni leen publicaciones especializadas; todos estos aspectos esenciales para su desempeño docente (Hernández, 2015; Rojas, 2008). Como consecuencia estarían brindando estos docentes una formación sumamente básica y meramente repetitiva. Así tenemos que, en un estudio con profesores universitarios del Perú, se ha reportado que un amplio porcentaje de ellos no comprende lo que lee (Yarlenque et al., 2012). De tal modo que podemos preguntarnos ¿cómo se espera que profesores que no leen inculquen en sus estudiantes el hábito de lectura, más aún si se trata de fuentes especializadas? Obviamente la formación de los profesores de psicología debe ser reformulada en consonancia con una mayor exigencia académica y producción científica (Gómez, 2013).

El 9 de julio de 2014 se promulgó la Ley Universitaria $N^{\circ} 30220$, de actual vigencia y que pone más énfasis en la investigación para elevar la calidad académica en las universidades del país. Todo ello en correspondencia con la implementación de sistemas de licenciamiento de universidades y acreditación de 
programas académicos en diversos países de Latinoamérica (Di Doménico y Risueño, 2013). Pero, además, haciendo eco de una variedad de informes que años precedentes daban cuenta de la situación en que funcionaban las universidades peruanas; los mismos que indicaban que los aspectos más deficitarios se focalizaban en la investigación científica, la gestión administrativa y el perfil académico de los docentes (Asamblea Nacional de Rectores, 2004; Ministerio de Educación, 2006; Piscoya, 2006, 2011; Sime, 2001).

En tal sentido, si bien la nueva Ley Universitaria ha favorecido un proceso de reforma que ha permitido mejorar los estándares académicos alineándolos con criterios internacionales más exigentes, tanto en su forma como en el contenido (Mejía et al., 2020), en cuanto a honestidad académica y productividad científica (López et al., 2020). Sin embargo, en el caso de la carrera de psicología, y sobre todo en las universidades de provincia, se evidencia una brecha entre los modelos formativos de otros países de la región; y en donde sus profesores son especialistas con una reconocida productividad académica (Klappenbach, 2003). Así pues, un estudio reciente en la ciudad de Arequipa, revela que los profesores de psicología de varias universidades, del ámbito público como el privado, no se sienten capacitados para emprender actividades investigativas a pesar de que ejercen la docencia universitaria con varios años de servicio (Arias et al., 2021).

En consecuencia, el presente estudio pretende abordar el tema de la lectura académica en los profesores de psicología y en la consideración de que este fenómeno está vinculado con su práctica docente y su producción científica. Por tanto, el objetivo del presente estudio fue analizar descriptivamente las fuentes académicas (libros y revistas científicas) que los profesores de psicología de una universidad privada de Arequipa han consultado o revisan de manera frecuente para preparar sus cátedras o mantenerse actualizados.

\section{Método}

\section{Diseño de investigación}

Se trata de una investigación cuantitativa con un diseño de investigación descriptivo transversal.

\section{Muestra}

La muestra estuvo constituida por 28 profesores de una universidad privada de la ciudad de Arequipa, con una edad promedio de 38.6 años y una desviación estándar de \pm 11.87 dentro de un rango de edad de 25 a 69 años de edad. El 35.7\% fueron varones y el $64.3 \%$ mujeres. En cuanto a su grado académico, el $21.4 \%$ eran bachilleres titulados, el 53.6\% tenían el grado de Magíster y el $25 \%$ el grado de Doctor. Con respecto al estado civil, el $35.7 \%$ eran casados y el $64.3 \%$ solteros, la media de hijos fue de 0.7 dentro de un rango de 0 a 3. El 60.7\% no tenía hijos, el $10.7 \%$ un hijo, el $25 \%$ dos hijos y el $3.6 \%$ tres hijos. Asimismo, el $67.9 \%$ no se dedica a investigar y el $32.1 \%$ es investigador acreditado por CONCYTEC o bien tiene experiencia en investigación y ha publicado algunos artículos. Los profesores fueron seleccionados de forma no probabilística mediante la técnica de grupos intactos.

\section{Instrumento}

Se aplicó una ficha de datos que recaba información sobre el sexo, la edad, el grado académico, el estado civil, el número de hijos, si tiene experiencia en investigación y los libros que los participantes habían leído en los últimos seis meses con los espacios correspondientes para colocar los datos bibliográficos referenciales. Asimismo, se consulta sobre las tres revistas científicas que los participantes conocen y consultan frecuentemente con espacios para colocar datos como el nombre de la revista, la institución que la edita, el país y la temática de la publicación. Finalmente, en el caso de los participantes que no respondieron este último segmento, se les pide escoger una de las seis opciones siguientes: 1) no tengo tiempo para leer, 2) mi presupuesto no es suficiente para comprar libros o revistas, 3) estoy 
ocupado con otras obligaciones, 4) nunca he leído revistas científicas especializadas, 5) es muy difícil acceder a las revistas especializadas, y 6 ) las revistas especializadas se publican en idiomas que no comprendo.

\section{Procedimiento}

Todos los participantes accedieron a participar voluntariamente y dieron su consentimiento. Asimismo, se contó con la aprobación del director de pregrado y del jefe de departamento del programa profesional de psicología de la universidad de la que provenían los participantes. La aplicación de los instrumentos tuvo lugar de forma grupal en una sola ocasión, en el mes de noviembre del año 2019.

\section{Resultados}

La media de libros leídos en los últimos seis meses antes de la aplicación de la ficha de datos fue de 3.64 (D.E.=1.28), de modo que el 3.6\% $(n=1)$ indicó que no había leído ningún libro, el 14.3\% $(n=4)$ leyó dos libros, el $25 \%(n=7)$ leyó tres libros, el otro $25 \%$ cuatro libros y el $32.1 \%(n=9)$ leyó cinco o más libros. Sin embargo, solo en dos casos, los participantes fueron capaces de completar la información bibliográfica de los libros leídos. Con respecto a las revistas especializadas, se obtuvo una media de 1.64 (D.E.=1.41), de manera que el $39.3 \%(n=11)$ indicó no conocer revistas científicas, el 3.6\% $(n=1)$ identificó una, el 10.7\% $(n=3)$ refirió dos, y el $46.4 \%(n=13)$ brindó los datos de tres revistas especializadas. Cabe resaltar que tres participantes indicaron como revistas a indexadoras tales como Dialnet, Redalyc y Elsevier, por tanto, se les ubicó en la categoría de no conocen revistas científicas. Además, las revistas académicas que fueron identificadas versan sobre psicología general, psicología educativa, familia, neuropsicología, psicología clínica y filosofía. La revista más referida fue Liberabit, que edita la Universidad San Martín de Porres en Perú desde el año 1995.

\section{Figura 1}

Motivos para no leer revistas científicas especializadas

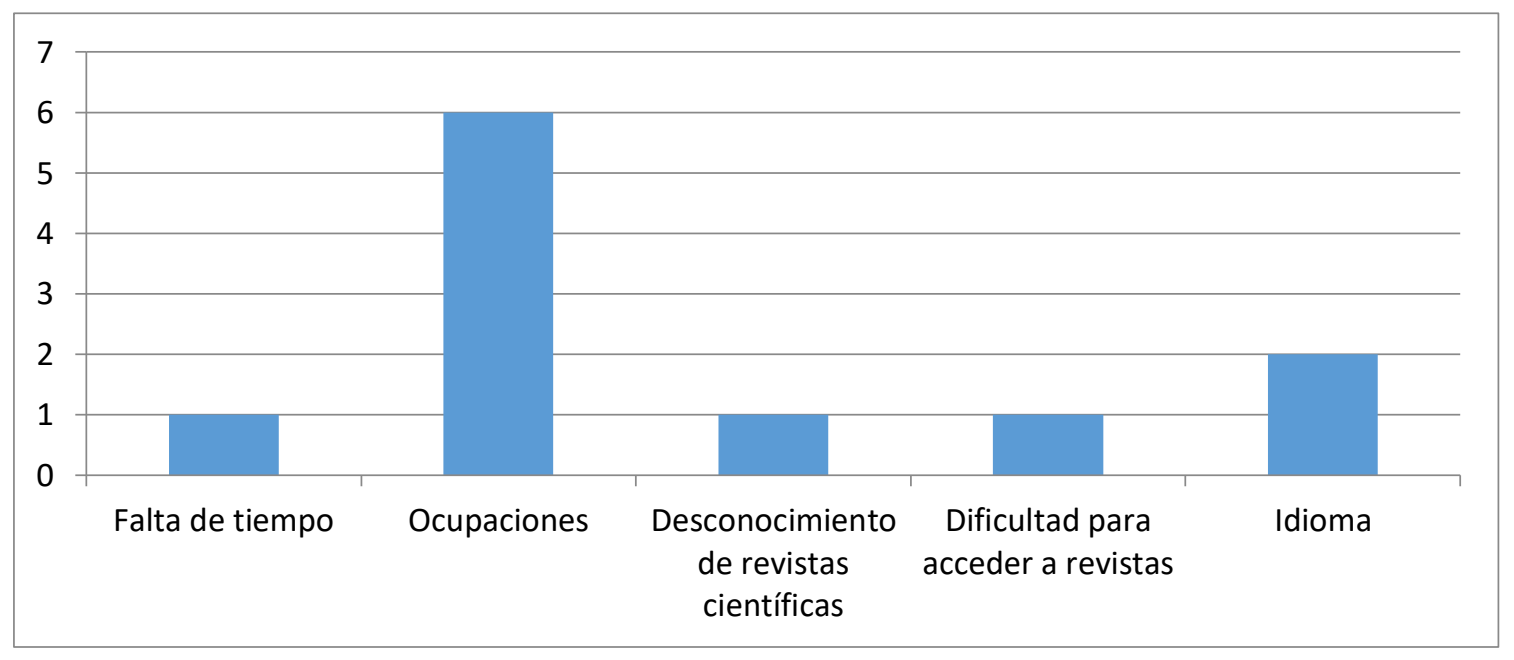

Por otro lado, frente a los motivos que se seleccionaron para no leer revistas especializadas, se tiene que seis participantes indicaron que tienen múltiples ocupaciones, dos indicaron que las revistas se 
publican en idiomas que no conocen y una persona señaló que, por falta de tiempo, al igual que por desconocimiento y dificultad para acceder a ellas.

Además, se realizaron cruces de datos con la prueba no paramétrica Chi Cuadrado en función de las variables sociodemográficas recogidas con la finalidad de detectar algunos patrones de lectura académica entre los participantes. De manera tal que, en cuanto al sexo, las mujeres leen más libros que los varones, con una media de 3.70 y 3.61, respectivamente; mientras que son los varones quienes conocen más revistas especializadas con una media de 2.40 y las mujeres tienen una media de 1.22; pero en ninguno de los casos las diferencias fueron significativas ( $\mathrm{p}=.058)$.

Por otro lado, tomando en cuenta el grado académico, se tiene que los participantes que poseen el grado de Magíster (44.4\%) leen más libros que quienes tienen grado de Bachiller (22.2\%) o Doctorado (33.3\%). No obstante, quienes tienen grado de Doctor identifican más revistas especializadas (71.4\%) a diferencia de quienes tienen el grado de Bachiller (33.3) o Magíster (40\%); aunque como en el caso anterior, las diferencias no fueron significativas ( $p>.05)$. En cuanto al estado civil, se tiene que los solteros (33.3\%) leen más libros que los casados (30\%), pero los casados (50\%) identifican más revistas especializadas que los solteros (44.4\%); sin embargo, no se registraron diferencias significativas ( $p>.05)$. Por otro lado, el tener o no tener hijos no reportó diferencias significativas en la lectura de libros $(\mathrm{p}=.836)$ ni en la identificación de revistas especializadas $(p=.730)$.

Finalmente, en cuanto a la experiencia en investigación, un $44.4 \%$ de profesores que investigan leen más libros que el $26.3 \%$ de quienes no investigan ( $p=.054$ ); y un $77.8 \%$ de los profesores con experiencia en investigación identificaron más revistas especializadas que el 31.6\% de los casos entre quienes no investigan, con diferencias significativas en el segundo caso $(p=.019)$.

\section{Discusión}

Los datos reportados en esta investigación aportan información relevante para comprender cómo se manifiesta la lectura académica entre los profesores de psicología de una universidad privada de la ciudad de Arequipa. En primer lugar, si bien la lectura de libros es una actividad muy difundida entre los participantes, un alto porcentaje de profesores (39.3\%) indicó no conocer revistas científicas especializadas; lo que supone que no mantendrían actualizados sus conocimientos ya que las mismas, por su periodicidad serial, permiten difundir información arbitrada producto de investigaciones científicas recientes (Hernández, 2015).

Por otro lado, entre los motivos que se indican para no leer o consultar revistas especializadas se tienen las múltiples ocupaciones, el idioma de publicación de las revistas, la falta de tiempo, el desconocimiento y la dificultad para acceder a ellas. Pero parece ser que todo confluye en la carencia de una cultura de investigación que abarca a la lectura académica (Rojas, 2008) ya que hasta un 93\% de las revistas científicas que se publican en América Latina son de acceso abierto, es decir, no cuesta descargar los artículos de sus plataformas virtuales y la mayoría de ellas se publican en español (Ramos-Galarza, 2018).

Un dato que refuerza lo anteriormente señalado es que, ni el sexo, ni el estado civil, el número de hijos, o el grado académico, mostraron diferencias significativas en cuanto a la lectura de libros o la identificación de revistas especializadas; más sí el tener experiencia en investigación o ser investigador acreditado. De modo que, el ser profesor investigador o haber publicado artículos científicos, sin necesariamente estar certificado como investigador, genera una ventaja académica entre los profesores en estudio.

Evidentemente, estos resultados no pueden generalizarse a otras casas de estudios del país debido al reducido tamaño de la muestra; pero si consideramos que los informes previos a la Ley 30220 sobre la situación académica de las universidades peruanas reportaban que hasta un $95 \%$ de los profesores universitarios del país no investiga (Asamblea Nacional de Rectores, 2004, 2012; Ministerio de Educación, 
2006) podemos colegir que la situación no ha cambiado mucho. Sin embargo, algunos reportes posteriores a la promulgación del mencionado marco legal, sugieren que las actividades investigativas en las universidades se han ampliado, dinamizado y formalizado a través de la constitución de grupos y centros de investigación; así como del establecimiento de criterios más objetivos para la evaluación y acreditación de los investigadores peruanos (Consejo Nacional de Ciencia, Tecnología e Innovación Tecnológica, 2016). De hecho, de las 143 universidades peruanas que funcionaban antes del 2014, solo 92 han conseguido licenciarse, siendo uno de los criterios más valorados, la producción científica.

Los resultados aquí reportados sugieren que aún falta mucho por hacer sobre todo en cuanto a la contratación de profesores universitarios con perfil académico basado en la producción científica, que tengan conocimientos actualizados y sean capaces de orientar académicamente a los estudiantes de psicología. En ese sentido, al ser la psicología una ciencia, se requiere de profesores altamente capacitados que brinden información basada en evidencias, con formación en investigación y que mantengan el hábito de la lectura académica como parte de su quehacer docente (Gómez, 2013; Klappenbach, 2003). De ahí, que respuestas como falta de tiempo o las múltiples ocupaciones que despliegan dentro de su ámbito profesional no son sino justificaciones infundadas pues nadie obliga al ingreso a la docencia universitaria (Arias, 2013); y si se asumen tales responsabilidades deben ser desplegadas con el mayor compromiso posible.

Se trata más bien de promover una cultura de meritocracia académica al interior de las universidades para garantizar estándares educativos adecuados a las jóvenes generaciones de estudiantes que serán los profesionales del mañana (Arias et al., 2021). Asimismo, tal parece ser, que existe una brecha entre el ámbito profesional y el ámbito académico en tanto se conciben como campos desligados uno del otro; pues son los profesores que investigan los que tienen una mayor cultura académica en comparación con quienes no investigan. Pero en realidad, sea que se investigue o no, un profesional debe mantenerse actualizado a través de publicaciones especializadas dentro de su respectivo campo de acción. No debe dejarse de lado este aspecto, y, muy por el contrario, debe promoverse la lectura de revistas especializadas entre profesores y estudiantes universitarios para ejercer los diversos aspectos de la psicología profesional con solvencia académica.

\section{Referencias}

Alarcón, R. (2002). Fuentes de felicidad: ¿Qué hace feliz a la gente? Revista de Psicología de la Pontificia Universidad Católica del Perú, 20(2), 169-196. https://doi.org/10.18800/psico.200202.001

Álvarez, D. M., Charvet, M. C., Espinoza, J. F., y Heredia, D. F. (2021). Formación del psicólogo: perspectivas en el contexto ecuatoriano. Desarrollo y Transformación Social, 10, 1-11. http://repositorio.uisek.edu.ec/handle/123456789/4187

Arias, W. L. (2013). Crisis de la universidad en el Perú: Un problema de su naturaleza e identidad. Educación, 19, 23-39. https://doi.org/10.33539/educacion.2013.n19.1017

Arias, W. L., y Justo, O. (2012). Velocidad de lectura e inteligencia en estudiantes de ingeniería. Revista de Psicología de la Universidad Católica San Pablo, 2, 43-55. https://revistas.ucsp.edu.pe/index.php/psicologia/article/view/9

Arias, W. L., Merino-Soto, C., Salas, E., Flores-Valdivia, G., y Zegarra-Valdivia, J. (2021). Motivaciones y dificultades percibidas para investigar: una aproximación exploratoria y no paramétrica en docentes de Psicología de Arequipa (Perú). (Artículo sometido a revisión).

Arias, W. L., Rivera, R., Ceballos, K., Maquera, C., Melgar, C., Sota, A., y Díaz Cano, M. (2018). Motivación de logro académico en estudiantes universitarios de Psicología: Un análisis psicométrico y comparativo de los datos. Revista Peruana de Investigación Educativa, 10, 178-189. https://doi.org/10.34236/rpie.v10i10.78 
Arias, W. L., Zegarra, J., y Justo, O. (2014). Estilos de aprendizaje y metacognición en estudiantes de $\begin{array}{llll}\text { Psicología de } \quad \text { 267-279. } & \text { 20(2), }\end{array}$ http://www.scielo.org.pe/scielo.php?script=sci_arttext\&pid=S1729-48272014000200008

Asamblea Nacional de Rectores (2004). Informe sobre educación superior universitaria. ANR.

Asamblea Nacional de Rectores (2012). Panorama de la investigación en la universidad peruana. ANR.

Carrillo, S., y Cuenca, R. (Eds.). (2019). Vidas desiguales. Mujeres, relaciones de género y educación en el Perú. Instituto de Estudios Peruanos.

Cavallo, G., y Chartier, R. (Eds.) (2011). Historia de la lectura en el mundo occidental. Taurus.

Cisneros, M., Olave, G., y Rojas, I. (2013). Alfabetización académica y lectura inferencial. Ecoe Ediciones.

Consejo Nacional de Ciencia, Tecnología e Innovación Tecnológica (2016). I Censo Nacional de Investigación y Desarrollo a Centros de Investigación 2016. Concytec.

Deheane, S. (2015). Aprender a leer. Siglo Veintiuno Editores.

Di Doménico, C., y Risueño, A. (2013). Procesos de acreditación de carreras de psicología en Argentina. Estado actual y prospectiva. Integración Académica en Psicología, 1(2), 24-28. https://integracionacademica.org/anteriores/10-volumen-1-numero-2-2013/20-procesos-de-acreditacion-de-carrerasde-psicologia-en-argentina-estado-actual-y-prospectiva

Gómez, E. N. (2013). La formación de profesores en psicología. Integración Académica en Psicología, 1(2), 70-80. https://integracion-academica.org/vol1numero2-2013/24-la-formacion-de-profesores-enpsicologia

Guadalupe, C., y Villanueva, A. (2013). PISA 2009/2000 en América Latina: una relectura de los cambios en el desempeño lector y su relación con las condiciones sociales. Apuntes, 40(72), 157-192. http://hdl.handle.net/11354/487

Hernández, R. M. (2015). Proceso editorial de una revista científica: cumpliendo con los requisitos de publicación. Revista Peruana de Psicología y Trabajo Social, 4(1), 77-84. https://repositorio.usil.edu.pe/handle/usil/2612

Klappenbach, H. (2003). La globalización y la enseñanza de la Psicología en Argentina. Psicologia em Estudo, 8(2), 3-18. https://doi.org/10.1590/\$1413-73722003000200002

León, R. (2021). Literatura, lectura y escritura en tiempos de COVID. Universidad Ricardo Palma, Universidad Politécnica y Artística del Paraguay.

Ley $\mathrm{N}^{\circ} 30220$ de 2014. Ley Universitaria. El Peruano. 9 de julio de 2014. 527213-33.

López, D. M, Eraña, I. E., Segura-Azuara, N. A., Piedra, I. D., Díaz, J. A., y López, M. V. (2020). Percepciones de los profesores sobre la deshonestidad académica en estudiantes de Medicina: prevalencia, motivaciones e implicaciones. Educación Médica, 21(5), 285-291. https://doi.org/10.1016/j.edumed.2018.07.009

Mejía, C. R., Chacón, J. I., Garay, E., Jorge, M., Delgado-García, S., Aveiro, R., Pacheco-Barios, N., y Serrano, F. (2020). Capacitaciones e investigación realizados por los recursos humanos en salud, Latinoamérica. Educación Médica, 21(5), 292-298. https://doi.org/10.1016/j.edumed.2018.08.005

Merino-Soto, C., y Salas-Blas, E. (2016). Estructura de las motivaciones y dificultades percibidas para la investigación entre los docentes universitarios: estudio preliminar. Revista Interamericana de Psicología, 50(2), 161-169. https://doi.org/10.30849/rip/ijp.v50i2.34

Ministerio de Educación del Perú (2006). La universidad en el Perú. Informe 2006. Razones para una reforma universitaria. MINEDU.

Montes, I. (2012). Investigación longitudinal de los hábitos de estudio en una cohorte de alumnos universitarios. Revista Lasallista de Investigación, 9(1), 96-110. https://www.redalyc.org/pdf/695/69524955005.pdf

Piscoya, L. (2006). Ranking universitario en el Perú. ANR.

Piscoya, L. (2011). ¿A dónde nos llevan nuestras universidades? Universidad Inca Garcilaso de la Vega.

Pradelli, A. (2013). El sentido de la lectura. Paidós. 
Ramos-Galarza, C. (2018). Los mitos de la publicación de un artículo científico psicológico en Ecuador. $\begin{array}{llll}\text { Revista Chilena } & \text { 194-197. }\end{array}$ https://scielo.conicyt.cl/scielo.php?script=sci_arttext\&pid=S0717-92272018000300194

Rojas, L. R. (2008). ¿Por qué publicar artículos científicos? Orbis, 10(4), 120-137. https://www.revistaorbis.org/pdf/10/Doc1.pdf

Sime, L. (2001). Universidad y currículo. Pontificia Universidad Católica del Perú.

Velarde, E., y Canales, R. (2008). La lectura en el Perú: Drama y esperanza. Universidad Nacional de Educación Enrique Guzmán y Valle.

Yarlenque, L., Javier, L., Núñez, E., Navarro, L., Cerrón, A., y Monroe, J. (2012). Comprensión lectora en los docentes de Educación básica de la región Junín. Revista de Investigación en Psicología, 15(1), 8391. https://doi.org/10.15381/rinvp.v1511.3666 